\title{
Measuring Galactic Extinction: A Test
}

\section{Citation}

Arce, Héctor G., and Alyssa A. Goodman. 1999. "Measuring Galactic Extinction: A Test." The Astrophysical Journal 512 (2): L135-38. https://doi.org/10.1086/311885.

\section{Permanent link}

http://nrs.harvard.edu/urn-3:HUL.InstRepos:41397457

\section{Terms of Use}

This article was downloaded from Harvard University's DASH repository, and is made available under the terms and conditions applicable to Other Posted Material, as set forth at http:// nrs.harvard.edu/urn-3:HUL.InstRepos:dash.current.terms-of-use\#LAA

\section{Share Your Story}

The Harvard community has made this article openly available.

Please share how this access benefits you. Submit a story.

Accessibility 


\title{
MEASURING GALACTIC EXTINCTION: A TEST
}

\author{
Héctor G. Arce ${ }^{1}$ and Alyssa A. Goodman ${ }^{2}$ \\ Harvard-Smithsonian Center for Astrophysics, 60 Garden Street, Cambridge, MA 02138; harce@cfa.harvard.edu, agoodman@cfa.harvard.edu \\ Received 1998 November 13; accepted 1998 December 21; published 1999 January 14
}

\begin{abstract}
We test the recently published all-sky reddening map of Schlegel, Finkbeiner, \& Davis (hereafter SFD) using the extinction study of a region in the Taurus dark cloud complex by Arce \& Goodman (hereafter AG). In their study, AG use four different techniques to measure the amount and structure of the extinction toward Taurus, and all four techniques agree very well. Thus, we believe that the AG results are a truthful representation of the extinction in the region and can be used to test the reliability of the SFD reddening map. The results of our test show that the SFD all-sky reddening map, which is based on data from COBE/DIRBE and IRAS/ISSA, overestimates the reddening by a factor of 1.3-1.5 in regions of smooth extinction with $A_{V}>0.5$ mag. In some regions of steep extinction gradients, the SFD map underestimates the reddening value, probably because of its low spatial resolution. We expect that the astronomical community will be using the SFD reddening map extensively. We offer this Letter as a cautionary note about using the SFD map in regions of high extinction $\left(A_{V}>0.5 \mathrm{mag}\right)$, since it might not be giving accurate reddening values there.
\end{abstract}

Subject headings: dust, extinction — infrared: ISM: continuum

\section{INTRODUCTION}

Extinction and reddening caused by interstellar dust effects the detected emission from most astronomical sources in the sky. In most Galactic and extragalactic studies, especially when studying so-called "standard candles," the effects of dust on the source's detected brightness and color need to be taken into account. Hence, it is very desirable to know the extinction (and reddening) anywhere on the sky.

Recently, Schlegel, Finkbeiner, \& Davis (1998, hereafter SFD) published an all-sky reddening map, based on satellite observations of far-infrared emission (at 100 and $240 \mu \mathrm{m}$ ) from dust. This new reddening map will surely be used by many researchers seeking to apply reddening "corrections" to their work, so it is very important to verify its accuracy and reliability. The SFD map uses data obtained by the DIRBE (Diffuse InfraRed Background Experiment) on board $C O B E$ combined with the ISSA (IRAS Sky Survey Atlas) images. The COBE/ DIRBE experiment had better control of absolute calibration than did IRAS, but it had a larger beam $(0.7$, as compared with $\sim 5^{\prime}$ for $I R A S$ ). SFD use the DIRBE data to calibrate the IRAS/ ISSA images, and, after sophisticated processing, they obtain a full sky map of the $100 \mu \mathrm{m}$ emission from dust, with point sources and the zodiacal light foreground removed. ${ }^{3}$ Their resulting reddening map is more accurate and has better resolution $(\sim 6$ '. 1$)$ than the previous existing all-sky reddening map of Burstein \& Heiles (1978, 1982).

In order to test the extinction map derived from the SFD analysis, we compare it with Arce \& Goodman's (1998, hereafter AG) recent extinction study of a region of the Taurus dark cloud complex. The AG study uses four different techniques to measure extinction along two constant right ascension "cuts" of several degrees in length. The four techniques in AG utilize (1) the color excess of background stars for which spectral types are known; (2) the ISSA 60 and $100 \mu$ m images; (3) star counts; and (4) an optical ( $V$ and $R$ ) version of the average

\footnotetext{
${ }^{1}$ National Science Foundation Minority Graduate Fellow.

${ }^{2}$ National Science Foundation Young Investigator.

${ }^{3}$ See SFD for a full description of their elaborate foreground zodiacal light subtraction technique, point-source extraction, and overall data reduction.
}

color excess method used by Lada et al. (1994). The study finds that all four methods give generally similar results and concludes that all four techniques are reliable ways to measure extinction in regions where $A_{V} \lesssim 4 \mathrm{mag}$. In this Letter, we compare the extinction map derived from SFD with the AG extinction map that is based on ISSA 60 and $100 \mu \mathrm{m}$ images in the Taurus region. This comparison provides a test of the reliability of the SFD reddening map for regions of extinction $\left(A_{V}\right)$ higher than $0.5 \mathrm{mag}$.

\section{COMPARING THE TWO RESULTS}

In this section, we compare the extinction derived from the SFD reddening map (hereafter $A_{V_{\mathrm{SFD}}}$ ) with the extinction $\mathrm{AG}$ obtained using the ISSA 60 and $100 \mu \mathrm{m}$ maps (hereafter $\left.A_{V_{\text {ISSA }}}\right)$. In order to be consistent, we must compare both methods with the same spatial resolution. As part of their image reduction procedure, SFD convolve the ISSA images with a FWHM $=3 ! 2$ Gaussian, which results in ISSA images with 6!.1 resolution. For the purpose of comparing the two extinction maps, we also convolve the AG ISSA images with a FWHM $=3$ '.2 Gaussian, so that both extinction determinations have the same resolution.

When AG compare $A_{V_{\text {ISSA }}}$ with the extinction measured with star-counting techniques and the average color excess method (techniques 3 and 4 above), they do so by plotting extinction versus declination. The extinction values shown in Figures 3 and 5 of AG are actually traces of the extinction averaged over the $\sim 10^{\prime}$ span (in the right ascension direction) of their CCD fields. For consistency, we consider the same kind of average, and we obtain the SFD reddening $\left[E(B-V)_{\mathrm{SFD}}\right]$ for the two $10^{\prime}$-wide constant right ascension cuts of $\mathrm{AG}$ and the average $E(B-V)_{\mathrm{SFD}}$ over right ascension. In order to convert from the color excess value given by the SFD maps to visual extinction, we use the equation $A_{V}=R_{V} E(B-V)$, where $R_{V}$, the ratio of total to selective extinction, is equal to 3.1 (SFD; Kenyon, Dobrzycka, \& Hartman 1994; Vrba \& Rydgren 1985).

In Figure 1, we plot extinction versus declination for the two constant right ascension cuts in the Taurus dark cloud complex region. In addition to $A_{V_{\mathrm{SFD}}}$ and $A_{V_{\mathrm{ISSA}}}$, we show the extinction traces of the extinction obtained from the average color excess 

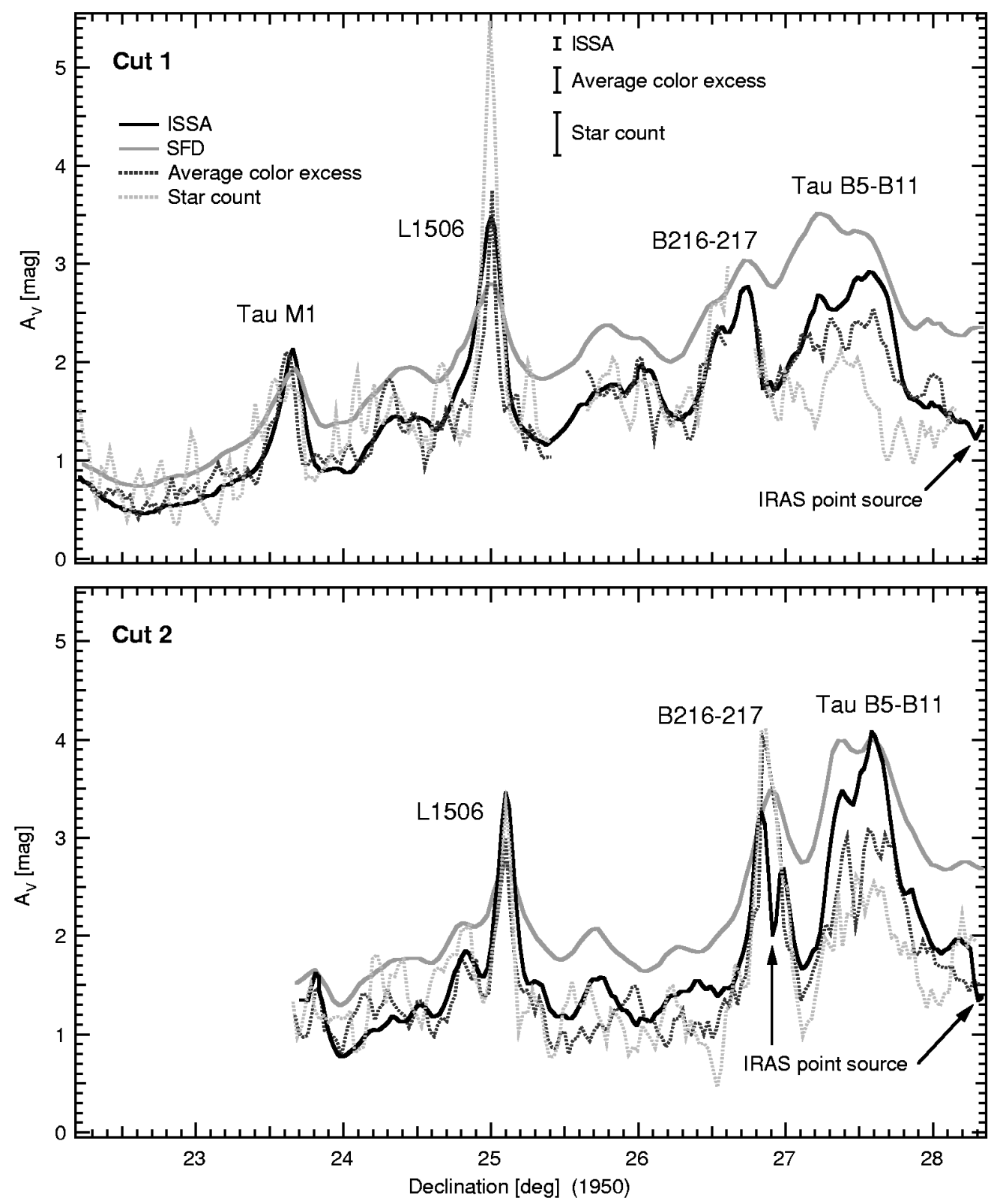

FIG. 1.-Plots of extinction vs. declination for cut 1 and cut 2 of AG. The first cut is centered on R.A. $=4^{\mathrm{h}} 19^{\mathrm{m}} 33^{\mathrm{s}}$ (B1950), and the second cut is centered on R.A. $=4^{\mathrm{h}} 18^{\mathrm{m}} 24^{\mathrm{s}}(\mathrm{B} 1950)$. The gray solid line is the extinction obtained from the SFD reddening map. The dark solid line is the extinction obtained using the ISSA 60 and $100 \mu \mathrm{m}$ images, the gray dotted line is the extinction from star counts, and the dark dotted line is the average color excess technique from AG. The average $1 \sigma$ random error for each of AG's extinction methods is shown. The peaks in extinction that are associated with named dark clouds and IRAS cores are identified. Dips in the $A_{V_{I S S A}}$ trace, due to artificial drops in the extinction caused by the presence of IRAS point source in the images, are also shown.

and star count methods of AG, for reference. We will concentrate our discussion on the ISSA (AG) and SFD methods. It can be seen that these two methods trace similar structure in both cuts. The extinction peaks associated with the IRAS core Tau M1 (Wood, Myers, \& Daugherty 1994), the dark cloud L1506, the dark cloud B216-217, and the IRAS cores Tau B5 and Tau B11 (Wood et al. 1994) are easily detected. The $1 \sigma$ error (not including systematics) of $A_{V_{\mathrm{ISSA}}}$ is $0.12 \mathrm{mag}$ (AG). SFD quote an uncertainty of $16 \%$ in their reddening estimates. For the most part, in regions unassociated with a pronounced peak in extinction, $A_{V_{\mathrm{SSD}}}$ is about a factor of 1.3-1.5 larger than $A_{V_{\mathrm{ISSA}}}$. It is also important to note that in regions where there is a peak in the extinction, the value of $A_{V_{\text {ISSA }}}$ is closer to that of $A_{V_{\mathrm{SFD}}}$ than in the rest of the trace, and in some of the peaks, $A_{V_{\mathrm{ISSA}}}$ exceeds $A_{V_{\mathrm{SFD}}}$.
There are two places where the ISSA and SFD traces appear to show very different extinction structures. In both of these places, $A_{V_{\text {ISA }}}$ shows a steep dip: one is near the peak associated with B216-217 in cut 2 (around decl. $=26^{\circ} 9$ ), and the other is near the end of the cut (around decl. = 28.3) (see Fig. 1). These two regions coincide with the position of IRAS point sources and will be discussed in more detail further on.

\section{COMPARISON BETWEEN THE TWO METHODS}

SFD use data from the IRAS and DIRBE experiments to construct a full-sky map of the Galactic dust based on its farinfrared emission. The IRAS data are used as a source of 100 $\mu \mathrm{m}$ flux images, and the DIRBE data are used for absolute calibration and as a source of $240 \mu \mathrm{m}$ data. SFD derive the 
dust color temperature using the ratio of 100 to $240 \mu \mathrm{m}$ emission from DIRBE, ${ }^{4}$ and then they use this dust color temperature to convert their ISSA-based maps of $100 \mu \mathrm{m}$ emission to maps of dust column density. As a result of this procedure, the SFD ISSA-based $100 \mu \mathrm{m}$ emission map has a spatial resolution of $6 ! 1$, but their dust color temperature map has a resolution of $\sim 1.4$ (D. J. Schlegel 1998, private communication).

To transform from the map proportional to dust column (hereafter $\left.\tau_{\mathrm{SFD}}\right)$ to a reddening $[E(B-V)]$ map, SFD use the correlation between the intrinsic $B-V$ color of elliptical galaxies and the $\mathrm{Mg}_{2}$ line strength. The $\mathrm{Mg}_{2}$ line strength of an elliptical galaxy correlates well with its intrinsic $B-V$ color, so that the $\mathrm{Mg}_{2}$ line index can be used along with photometric measurements of the galaxy in order to obtain a reasonably accurate measurement of its reddening (Faber et al. 1989). SFD use a procedure where the residual of the $B-V$ color versus the $\mathrm{Mg}_{2}$ line index for 389 elliptical galaxies is correlated with the estimated reddening from their maps, using a SpearmanRank method. The resultant fit is then used by SFD to convert from $\tau_{\mathrm{SFD}}$ to reddening $\left[E(B-V)_{\mathrm{SFD}}\right]$ at each pixel. We then convert reddening to extinction, using the relation $A_{V_{\mathrm{SFD}}}=$ $R_{V} E(B-V)_{\mathrm{SFD}}$, with $R_{V}=3.1$ (SFD).

In order to obtain their ISSA-based extinction, $A_{V_{\mathrm{ISSA}}}, \mathrm{AG}$ begin by using ISSA 60 and $100 \mu \mathrm{m}$ images to obtain a dust color temperature map from the flux ratio at each pixel. This color temperature is then used to convert the observed $100 \mu \mathrm{m}$ flux to the $100 \mu \mathrm{m}$ optical depth, $\tau_{100}$. As in SFD, the next conversion, from dust opacity to extinction, is tied to a separate technique of obtaining extinction. AG chose to use a method similar to that described in Wood et al. (1994), which itself is ultimately based on work by Jarrett, Dickman, \& Herbst (1989). Jarrett et al. (1989) correlate a $60 \mu \mathrm{m}$ optical depth $\left(\tau_{60}\right)$ with extinction $\left(A_{V}\right)$ obtained from star counts, and Wood et al. (1994) multiply the Jarrett et al. (1989) $\tau_{60}$ values by $100 / 60$ to derive a conversion from $\tau_{100}$ to $A_{V}$, assuming low optical depth. Thus, using Wood et al.'s technique, AG's conversion of $\tau_{100}$ to $A_{V}$ ultimately rests on Jarrett et al.'s correlation of the $60 \mu \mathrm{m}$ optical depth with star counts. As described in AG, this $\tau_{60}-A_{V}$ correlation is very tight for $A_{V}<4 \mathrm{mag}$, so we ascribe very little of the uncertainty in $A_{V_{\mathrm{ISSA}}}$ to this conversion.

\section{THE CAUSE OF THE DISCREPANCY}

AG obtain the extinction toward the Taurus region using four different techniques. All four give similar results in terms of the absolute value and overall structure of the extinction. Most importantly, AG find that $A_{V_{\mathrm{ISSA}}}$ agrees well with extinction values obtained by measuring the color excess of background stars for which spectral types are known, which is the most direct and accurate way to measure reddening (see Fig. 2 in $A G)$. So the question now is as follows: why does the SFD method give extinctions that differ from the four determinations of extinction made by $\mathrm{AG}$ ?

Absolute values. - Although the data reduction and the zeropoint determination of the emission are very elaborate and accurate in the SFD method, the normalization of the reddening per unit flux density [conversion between $\tau_{\mathrm{SFD}}$ and $E(B-$ $\left.V)_{\mathrm{SFD}}\right]$ seems to overestimate the reddening in regions of high dust opacity. As explained above, SFD use the correlation be-

\footnotetext{
${ }^{4}$ Note that deriving the dust temperature from a long-wavelength color ratio, such as $100 / 240 \mu \mathrm{m}$, is superior to using the $60 / 100 \mu \mathrm{m}$ ratio, because the effects of both the point sources and the transient heating of small grains are minimized.
}

tween the intrinsic $B-V$ color and the $\mathrm{Mg}_{2}$ line index in elliptical galaxies to convert from dust column density to reddening. It can be seen in Figure 6 of SFD that $90 \%$ of the 389 elliptical galaxies they use for the $B-V$ versus $\mathrm{Mg}_{2}$ regression have $E(B-V)_{\mathrm{SFD}}$ values of less than $0.1 \mathrm{mag}$. Moreover, it is clear from their Figure 6 that the fit is excellent for values of $E(B-V)_{\mathrm{SFD}}$ less than $0.15 \mathrm{mag}$, but for $E(B-V)_{\mathrm{SFD}}>0.15$ mag, the fit starts to diverge for the few points that exist. Galaxies with $E(B-V)_{\mathrm{SFD}}>0.15 \mathrm{mag}\left(A_{V}>0.5 \mathrm{mag}\right)$ seem to follow a trend leading to reddening values in regions with high dust opacity being overestimated. SFD state that the slight trend in the residual is not statistically significant, but that may be due to the fact that there are so few points with $E(B-$ $V)_{\mathrm{SFD}}>0.15 \mathrm{mag}$.

Figure 6 in SFD shows only two points (galaxies) that have $E(B-V)_{\mathrm{SFD}}>0.3$ mag. We use the point with $E(B-$ $V)_{\mathrm{SFD}}=0.32 \mathrm{mag}$ to asses the amount of overestimation by the SFD method in high reddening regions. Using Figure 6 in SFD and $A_{V}=R_{V} E(B-V)$, with $R_{V}=3.1$, for an extinction of around $1 \mathrm{mag}$, the SFD method would overestimate the extinction, at $1.31 \mathrm{mag}$. In fact, by comparing $A_{V_{\mathrm{SFD}}}$ with $A_{V_{\mathrm{ISSA}}}$, we find that the overestimation by SFD can be even more than that: when $A_{V_{\mathrm{ISSA}}}$ is $1, A_{V_{\mathrm{SFD}}}$ is typically 1.5. Thus, we are convinced that $S F D$ overestimates the reddening value to lines of sight where the extinction is more than $0.5 \mathrm{mag}$. Such overestimation is due to the fact that in the sample of 389 galaxies, most of them have $E(B-V)<0.1$ and very few have $E(B-V)>0.15$. This results in an accurate conversion between $100 \mu \mathrm{m}$ emission and reddening for regions with very low extinction $[E(B-V)<0.1]$ but a less accurate conversion where $E(B-V)>0.15\left(A_{V}>0.5 \mathrm{mag}\right)$.

One could argue that the SFD method overestimates the extinction because we overestimate the value of $R_{V}$ needed to convert $E(B-V)$ to $A_{V}$. We believe that is not so because several independent studies indicate that the value of $R_{V}$ in the Taurus dark cloud complex is around 3.1 and that it stays constant through the region for lines of sight in which $A_{V}<$ 3 mag (Kenyon et al. 1994; Vrba \& Rydgren 1985). Furthermore, $R_{V}=3.1$ gives very good agreement between $A_{V_{\text {ISSA }}}$ and extinction obtained using the color excess of background stars for which spectral types are known (AG).

Structure.-In $\S 2$, we noted that there are certain places where the traces of $A_{V_{\mathrm{ISSA}}}$ and $A_{V_{\mathrm{SFD}}}$ differ not only in value but also in structure. The most dramatic "structural" differences correspond to places where the cuts in Figure 1 intercept IRAS point sources. SFD remove point sources from the ISSA images before they use them, while AG do not. Both AG and SFD assume a single dust temperature for each line of sight. In AG's determination of $A_{V_{\text {ISSA }}}$, the high $60 \mu \mathrm{m}$ flux from IRAS point sources transforms into dust with high color temperature, which in turn causes an artificial dip in the extinction (see Wood et al. 1994 and references therein for more on this effect). We can see that SFD have done a good job at removing point sources by noticing that where $A_{V_{\text {ISSA }}}$ has artificial dips, $A_{V_{\text {SFD }}}$ traces the real structure (also traced by the other methods in AG). Thus, $A_{V_{\mathrm{SFD}}}$, unlike $A_{V_{\mathrm{ISSA}}}$, is free of any unphysical extinction due to IRAS point sources.

Less dramatic, but more curious, structural discrepancies between the two methods are seen near the extinction peaks in Figure 1. Although $A_{V_{\mathrm{SFD}}}$ is in general larger than $A_{V_{\mathrm{ISSA}}}$ by a factor of 1.3-1.5, it is important to realize that in some peaks, $A_{V_{\mathrm{SFD}}}$ is larger by a factor less than 1.3 and that in others, it is smaller than $A_{V_{\mathrm{ISSA}}}$. In addition, the Gaussian-looking extinction peaks traced by $A_{V_{\mathrm{SFD}}}$ are wider than those traced by $A_{V_{\mathrm{ISSA}}}$. We 
fitted Gaussians to the peaks associated with Tau M1 and L1506 for both traces in cut 1 , and we find that both peaks traced by $A_{V_{\mathrm{SFD}}}$ have FWHM wider than the peaks traced by $A_{V_{\mathrm{ISSA}}}$ by a factor of 1.3. This suggests that in regions of steep extinction gradients, the reddening obtained from the SFD map has a lower resolution than the quoted 6'.1.

We believe that this "smearing" in the SFD maps is caused by the difference in resolution between the ISSA flux maps (6'.1) and the COBE-limited color temperature maps (1.4) employed by SFD. In Figure 2, we plot the $100 \mu \mathrm{m}$ emission $\left(I_{100}\right)$ versus declination for a section of cut 1 , where the peaks in $I_{100}$ associated with Tau M1 and L1506 are clearly seen. The plot shows the traces of $I_{100}$ obtained by SFD $\left(I_{100_{\text {SFD }}}\right)$ and by $\operatorname{AG}\left(I_{100_{\text {ISSA }}}\right)$ averaged over the $\sim 10^{\prime}$ width (in right ascension) of the cut. It can clearly be seen that both traces are essentially equal and that the peaks have the same width and height. Thus, it is after both the dust color temperature and $I_{100}$ have been used together, in order to obtain the extinction, that the SFD method loses spatial resolution. Regions of steep extinction gradients are likely to have temperature gradients, but if the spatial resolution in the dust color temperature is $\sim 1.4$, then it is unlikely that these gradients would be detected. If the temperature gradients are not accounted for when calculating reddening, the result is areas with lower effective resolution. Therefore, the fact that SFD use a dust color temperature map with a spatial resolution a factor of 14 larger than the $I_{100}$ map results in a reddening map that does not have a constant spatial resolution of 6'1. Unfortunately, the DIRBE instrument has a limiting resolution of $\sim 1^{\circ}$, so it is not possible to do better with data taken with that instrument.

\section{CONCLUSION}

We test the COBE/IRAS all-sky reddening map of SFD using the extinction study of AG. In their study, AG use four different techniques to study the extinction in a region of the Taurus dark cloud complex. All four techniques give very similar results in terms of the value of $A_{V}$ and the structure in the extinction. Thus, the results of AG can be considered a truthful representation of the extinction in this region and can be used to test the reliability of the SFD reddening map.

We compare the extinction obtained by AG using ISSA 60 and $100 \mu \mathrm{m}$ images with the SFD reddening map. Our results show that, in general, the SFD method gives extinction values a factor of 1.3-1.5 larger than the extinction obtained by AG. We conclude that SFD overestimates the reddening value to lines of sight where $A_{V}>0.5 \mathrm{mag}$. We expect that this overestimation is caused by the fact that in the sample of 389

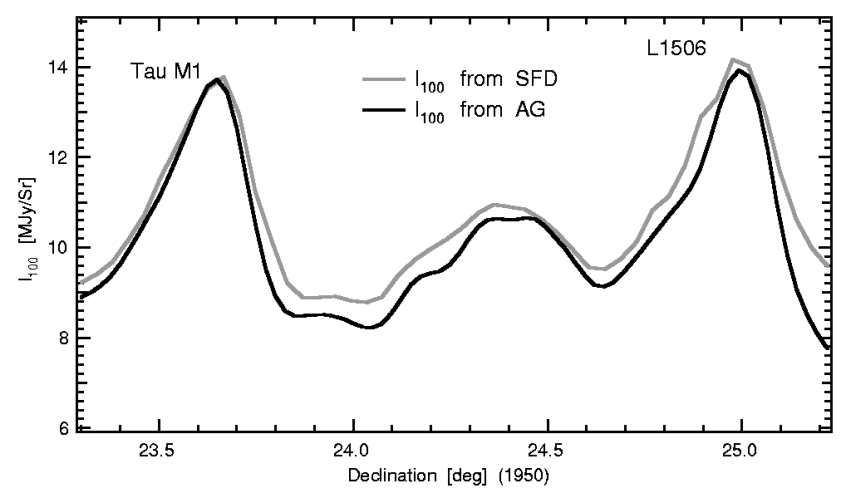

FIG. 2.-A plot of the $100 \mu \mathrm{m}$ dust emission vs. declination for a part of cut 1 . The light line is the emission obtained from the SFD $100 \mu \mathrm{m}$ map. The gray line is the emission from the AG $100 \mu \mathrm{m}$ map. The rise in the $100 \mu \mathrm{m}$ emission associated with Tau M1 and L1506 is identified.

galaxies used to calculate a conversion from dust column density to $E(B-V), 90 \%$ of the galaxies have low-reddening values $[E(B-V)<0.1]$ and very few $(\sim 4 \%)$ have highreddening $[E(B-V)>0.15]$ values. The lack of galaxies in high-reddening regions results in an inaccuracy in the conversion between dust column and reddening for lines of sight with $E(B-V)>0.15$. This bias should be studied in other regions of the sky in order to see how general this trend is. For now, we advise that caution be taken when using the SFD all-sky reddening map to obtain reddening (and extinction) values for regions with $E(B-V)>0.15\left(A_{V}>0.5 \mathrm{mag}\right)$, since the value given by SFD could be larger than the real value.

The behavior of $A_{V_{\mathrm{SFD}}}$ near steep gradients in extinction seems to be different from the overall "smoother" extinction regions. The difference between $A_{V_{\mathrm{SFD}}}$ and $A_{V_{\mathrm{ISSA}}}$ decreases near extinction peaks, and in some peaks, $A_{V_{\mathrm{SFD}}}$ even becomes smaller than $A_{V_{\text {ISSA }}}$. In addition, the peaks in $A_{V_{S F D}}$ are wider than the $A_{V_{\text {ISSA }}}$ peaks. We attribute this behavior to the fact that SFD use a dust temperature map with a spatial resolution of a factor of 14 larger than the $100 \mu \mathrm{m}$ intensity map used to obtain the reddening map. The poor resolution in the temperature maps results in a reddening map that shows less spatial resolution near extinction peaks.

We thank David Schlegel, Douglas Finkbeiner, and Mark Davis for their very useful comments on this work and Krzysztof Stanek for pointing out the SFD paper to us and for sharing his work on this topic. We also thank the referee for prompt and very helpful comments.

\section{REFERENCES}

Arce, H. G., \& Goodman, A. A. 1998, ApJ, submitted (AG)

Burstein, D., \& Heiles, C. 1978, ApJ, 225, 40 1982, AJ, 87, 1165

Faber, S. M., Wegner, G., Burstein, D., Davies, R. L., Dressler, A., LyndenBell, D., \& Terlevich, R. J. 1989, ApJS, 69, 763

Jarrett, T. H., Dickman, R. L., \& Herbst, W. 1989, ApJ, 345, 881
Kenyon, S. J., Dobrzycka, D., \& Hartman, L. 1994, AJ, 108, 1872

Lada, C. J., Lada, E. A., Clemens, D. P., \& Bally, J. 1994, ApJ, 429, 694

Schlegel, D. J., Finkbeiner, D. P., \& Davis, M. 1998, ApJ, 500, 525 (SFD)

Vrba, F. J., \& Rydgren, A. E. 1985, AJ, 90, 1490

Wood, D., Myers, P. C., \& Daugherty D. A. 1994, ApJS, 95, 457 